\title{
L'ethnologue dans les réseaux économiques des femmes migrantes : modes de présence simultanée entre la France et l'Afrique
}

An Ethnologist in the Economic Networks of Migrant Women: Simultaneous Ways of Moving between France and Africa

Una etnóloga en las redes económicas de mujeres migrantes: modos de presencia simultánea entre Francia y África

Jeanne Semin

\section{OpenEdition}

\section{Journals}

Édition électronique

URL : https://journals.openedition.org/remi/4989

DOI : 10.4000/remi.4989

ISSN : $1777-5418$

Éditeur

Université de Poitiers

Édition imprimée

Date de publication : 1 décembre 2009

Pagination : 101-113

ISBN : 978-2-911627-53-8

ISSN : 0765-0752

Référence électronique

Jeanne Semin, «L'ethnologue dans les réseaux économiques des femmes migrantes : modes de présence simultanée entre la France et l'Afrique », Revue européenne des migrations internationales [En ligne], vol. 25 - n³ | 2009, mis en ligne le 01 décembre 2012, consulté le 15 avril 2022. URL : http:// journals.openedition.org/remi/4989; DOI : https://doi.org/10.4000/remi.4989 


\title{
L'ethnologue dans les réseaux économiques des femmes migrantes : modes de présence simultanée entre la France et l'Afrique
}

\author{
Jeanne SEMIN*
}

\section{INTRODUCTION}

Le foisonnement des études et des discours actuels dans le domaine des migrations et des transferts transnationaux mobilise différentes sciences sociales : économie, géographie, sociologie, démographie, histoire, anthropologie, etc. Il n'est pas inutile dans ce contexte scientifique pluridisciplinaire de reparler de méthodologie, d'observations et d'analyses propres à qualifier ces phénomènes particuliers et leurs implications. Il sera ici question de la méthode de l'observation en plusieurs lieux, dite multi sited ethnography, que George Marcus considérait comme ideologically antidisciplinary (1995 : 97), et bien souvent mal définie et perçue comme un renouvellement méthodologique global appliqué à l'observation des sociétés post-coloniales. Elle s'appuie pourtant sur la tradition de l'observation ethnographique, définie et utilisée essentiellement par les ethnologues et anthropologues, bien qu'elle ne soit pas à l'usage exclusif de ceux-ci.

Lorsqu'on a pour sujet d'étude la migration, ou plus précisément une pratique sociale quelconque, économique ou culturelle, d'un groupe migrant, il est aujourd'hui tentant de poursuivre ses racines, ses " branchements », et ses retombées déterritorialisées dans plusieurs localités, ce qui invite à un " travail de terrain éclaté » (Amselle, 2001 : 7). Or l'ethnographie appliquée aux études migratoires, n'a pas vraiment fait, en France, l'objet de discussions méthodologiques sur les modes d'observation, ces dernières ayant plutôt porté récemment sur la réflexivité méthodologique et éthique des chercheurs (Ghassarian, 2002 ; Leservoisier, 2005), tout aussi importante. Ainsi les recherches dites multi situées se multiplient (Kane, 2001 ; Boesen et Marfaing, 2007 ; Dia, 2008) sans

\footnotetext{
* Doctorante, allocataire de la région d'Ile-de-France, Centre d'études des mondes africains, CNRS, 27 rue Paul Bert, 94204 Ivry-sur-Seine ; jeannesemin@hotmail.com
} 
pour autant que l'on s'interroge sur les avantages et les difficultés pratiques d'une telle méthode. Je proposerai donc une discussion épistémologique sur cette ethnographie multi située appliquée à l'observation des modes de vie des migrants d'Afrique Noire, entre ici et là-bas. Que nous apporte en réalité cette poursuite effrénée au fil des réseaux de connexions migratoires ? Peut-on parler systématiquement d'ethnographie multi située ? Comment construire son objet d'étude dans la multi localité ? Comment observer entre les sites une interaction significative ? Il s'agit ici d'ébaucher quelques réponses à ces questions de méthodologie à partir des textes fondateurs, majoritairement américains, de l'ethnographie multi située, des expériences françaises de cette méthodologie dans le domaine des études migratoires, et de notre expérience particulière d'une recherche sur la pratique des tontines par les femmes d'Afrique de l'Ouest. Mes recherches consistent en effet à décrire un système de relations vécues, sans cesse actualisé et redéfini par les échanges tontiniers et cérémoniels des femmes en Afrique et en France.

\section{ENJEUX ET PERSPECTIVES D’UNE MÉTHODE MULTI SITUÉE}

L'ethnographie multi située à proprement parler est née aux États-Unis dans un contexte académique et idéologique post-colonial. Les chercheurs en sciences humaines, en particulier Georges Marcus et Arjun Appaduraï, ont proposé un renouvellement méthodologique visant à saisir la réalité déterritorialisée des phénomènes sociaux de la « globalisation ». Il est bon de rappeler pourtant que ce mouvement dit de « globalisation », souvent considéré comme radicalement nouveau, s'inscrit dans une continuité. Les migrations collectives et individuelles ont de fait toujours existé. Des dispositifs antérieurs de " globalisation », entendue comme l'intégration de sociétés séparées dans un espace partagé au regard d'une dimension commune, ont ainsi favorisé l'échange entre les clans et les peuples et l'émergence de référents culturels communs : on peut en prendre pour exemple les échanges de kula dans l'aire Pacifique ou l'islamisation pour l'Afrique de l'Ouest. Mais les dispositifs contemporains de globalisation ont partie liée à l'utilisation de moyens technologiques (télévisions, téléphones portables, Internet, banalisation des transports aériens, etc.) permettant une réduction de la distance réelle et symbolique entre les hommes. Pour caractériser notre époque on peut donc aussi utiliser le terme technique et géopolitique d' " internationalisation », qui désigne : " l'utilisation d'un ensemble de techniques et de procédés réduisant l'espace-temps des échanges matériels informatifs et humains entre les territoires de la planète » (Dumont, 2007 : 22). Alors que, dans ce contexte, le phénomène des migrations se banalise, la fermeture des frontières intervient comme une réaction protectionniste des États du Nord. D’une migration baptisée « en noria $»^{2}$, du nom d'un système d'arrosage arabe par aller-retour de petits paniers successifs, permise jusqu'en 1970 par l'ouverture des frontières à la main-d'œuvre étrangère, on passe à une migration « en chaîne » ou en « réseau » : les migrants une fois installés, ne repartent plus si facilement et assurent plutôt la venue et le parrainage des parents et amis des communautés d'origine ${ }^{3}$.

2 Ce terme a été proposé et utilisé pour la première fois en 1954 par Robert Montagne dans ses travaux consacrés à la migration algérienne en France.

3 Ce point n'est pas spécifique aux migrations africaines; sur les migrations maghrébines et chinoises, voir les travaux d'Emmanuel Ma Mung. 
La mise en œuvre de réseaux efface en partie la notion de frontière et permet une grande souplesse répondant à la rigidité accrue des politiques migratoires des États européens. Les nouveaux outils de télécommunication facilitent l'interaction des membres d'une communauté internationale. Instantanément informés des événements importants concernant la reproduction de l'unité sociale, les migrants sont plus directement impliqués dans la vie de la famille ou du village d'origine. Les rapports sociaux ne sont plus ancrés dans une territorialité spatialisée. Si bien que l'absence n'est plus vécue de la même manière. La migration des Gens du Fleuve, par exemple, est devenue un phénomène semi autonome constitutif de l'histoire d'un peuple, et les migrations vers la France, si elles ont changé de forme ne se sont pas taries du fait de la fermeture des frontières. Cependant on assiste également à une diversification des lieux et réseaux d'immigration vers les ÉtatsUnis, l'Espagne et l'Italie, qui invite à parler d'une « multi-polarisation transnationale des villages » (Schmitz et Humery, 2008 : 69) de la Vallée du Fleuve Sénégal.

Les chercheurs en sciences humaines et en particulier en anthropologie tentent de se plier à cette fluidité, cette « liquidité » du monde (Bauman, 2007), d'y adapter leurs méthodes. Il n'est pas aisé pour le chercheur d'observer l'échange délocalisé, à défaut de se fluidifier soi-même, d'inventer la télé-transportation ou mieux encore d'acquérir au moins mentalement le don d'ubiquité. Cet échange est pourtant le vecteur d'un tissu social fondamental, qui peut paraître alors insaisissable. L'étude des migrations internationalisées a largement contribué à enrichir le lexique conceptuel permettant de décrire cette reconfiguration spatio-temporelle des rapports sociaux. En France, les rapports à l'espace et à la mobilité ont par exemple été repensés par Alain Tarrius à partir de la notion de « territoires circulatoires », faits de centralités multiples, et de la figure de l' « acteur migrant », créateur et reproducteur de liens sociaux au sein des diasporas. La notion de « territoire circulatoire » est le résultat de production de mémoires collectives et de pratiques d'échanges sans cesse plus amples (Tarrius, 1994). L’idée de diaspora, historiquement connotée comme subie et traumatique, a quant à elle fait place à la notion de «transnationalisme », définie comme « l'ensemble des processus par lesquels les immigrés tissent et entretiennent des relations sociales de nature multiple reliant leurs sociétés d'origine et d'accueil » (définition de Glick Schiller et al., traduite par Portes, 1999) C'est à travers des processus très variés (transferts monétaires, investissements immobiliers, communications téléphoniques, stratégies matrimoniales, etc.) que les migrants construisent des « communautés transnationales », ou à une plus petite échelle des « villages multi situés » (Dia, 2008). Le fait notamment d'envoyer régulièrement de l'argent et de participer à des projets sanitaires et sociaux communs dans le village d'origine est par exemple un moyen connu d'entretenir une forme de cohésion sociale au sein des communautés transnationales, de réunir ceux que l'espace éloigne.

Le rapport à l'espace social et relationnel des migrants est passé d'une « double absence » : ni ici, ni là-bas (Sayad, 1999) à l'expression d'un continuum entre les sociétés d'accueil et les sociétés d'origine « qui ne forment qu'une seule et même arène de l'action sociale » (Bretell, $\left.2000: 104-105^{4}\right)$. La simultanéité de la présence en plusieurs endroits reste cependant toujours difficile à observer. À titre d'exemple méthodologique et conceptuel, on peut citer la méthode dite d'analyse des réseaux sociaux, qui pourrait permettre

4 Citée et traduite pas William Berthomière et Marie-Antoinette Hily (2006). 
de se représenter graphiquement le fonctionnement en réseaux et l'interaction au sein des communautés transnationales et de les analyser. Utilisée principalement en histoire et en sociologie, un peu moins en anthropologie, où elle se cantonne au domaine de la parenté, la méthode sociographique est constitutive d'un champ épistémologique à part entière (Mercklé, 2008), et pourrait se révéler fort utile appliquée aux études migratoires. Elle ne peut cependant se suffire à elle-même, en ce qu'elle se borne à la graphie et la description d'interactions, qui, du fait qu'elles ne sont saisies que dans leur dimension comportementale, risquent alors d'être amputées du sens symbolique et affectif qui leur donne corps (Caillé, 2000 : 60) ; la nature réticulaire des liens et les déterminants de leur force restent alors à définir. Comment alors observer dans la multi localité ces interactions vécues qui se concrétisent par l'échange, matériel ou immatériel, et lient entre eux les membres des communautés transnationales?

\section{RÉSEAUX SOCIAUX ET ETHNOGRAPHIE : COMMENT OBSERVER LA SIMULTANÉITÉ ?}

On peut alors souligner l'apport spécifique que permet la méthode ethnographique, devenue multi située. Si, pendant des décennies, les ethnologues ont constitué leurs objets à partir de groupes localisés, aujourd'hui les anthropologues ne définissent plus la localité simplement par référence au territoire, et le " ethno », d'ethnographie prend donc un aspect réticulaire. L'observer peut impliquer le mouvement des chercheurs, mais observation et intégration restent les clefs méthodologiques de la discipline.

Les travaux d'Arjun Appaduraï, par exemple, nous renvoient à l'exigence méthodologique d'une ethnographie qui prenne en compte la complexité des trajectoires individuelles et collectives en multipliant les lieux d'investigation au fil des mouvements vécus. Le fait de suivre les migrants par-delà les frontières peut apparaître comme un exercice classique des études migratoires. Certaines expériences contemporaines de "mobilité participative » (Boesen et Marfaing, 2007 : 14), qui consiste à accompagner les migrants dans leurs déplacements, illustrent parfaitement la tension entre mobilité et installation au fondement des processus migratoires (Brachet, 2009). Mais l'originalité des études multi situées telles qu'elles sont définies par Marcus réside dans le fait de s'intéresser aux relations et interactions entre les différents sites de l'enquête. Ce sont ces dernières qui donnent sens à l'idée de communauté transnationale au-delà du mouvement des sujets d'un lieu à un autre (Marcus 1995 : 12). Comme pistes concrètes de cette ethnographie multi située, cet auteur énonçait d'ailleurs six modes possibles de construction d'un objet d'étude : suivre les individus dans leurs mobilités, suivre les objets (tracer la circulation d'un objet matériel d'étude), suivre la métaphore, la mobilité des signes, symboles et expressions, suivre les histoires, les allégories, leurs répercussions, résonances et redoublements déterritorialisés, suivre les vies ou les bibliographies, et enfin suivre les échos transnationaux de conflits familiaux ou ethniques (Marcus, 1995). Il s'agit, pour résumer, de suivre les informants et leur production culturelle où qu'ils aillent (Hylland Eriksen, 2003 : 5). Ne serait-il pas possible également d'ajouter à cette liste l'idée assez simple de suivre les pistes relationnelles des réseaux sociaux? 
Pour exemple, je propose d'aborder le processus de construction de ces relations d'appartenance à travers les tontines et échanges cérémoniels des femmes ouest africaines, immigrées en France. Mes enquêtes de terrain portant sur les tontines ont consisté en une observation de ces pratiques en plusieurs lieux. En effet, j'ai d'abord compris la méthode multi située comme un héritage de la méthode comparative, qui consiste à observer un même objet (les pratiques tontinières des femmes africaines) dans différents lieux au Mali et en France, avant de suivre l'invitation offerte par le terrain à suivre les connexions de relations et les réseaux de parenté entre ici et là-bas.

La perspective comparatiste avait tout d'abord pour objectif simple d'observer l'adaptation de la tontine, pratique d'échange bien connue en Afrique de l'Ouest, où elle est surtout féminine, à un contexte différent en France ${ }^{5}$. Mon sujet d'étude résidait dans l'hypothèse d'une continuité des pratiques tontinières des femmes ouest-africaines au-delà de la migration. Dans un premier temps, les personnes interviewées en France n'étaient pas liées à celles que je connaissais par ailleurs en Afrique. Je rencontrais au début de l'enquête en France d'énormes difficultés. Une entrée par l'espace public des associations s'est avérée impossible, s'agissant de l'usage que faisaient les migrantes des tontines. Celles-ci ne relèvent pas en France du cadre associatif, les réponses sur le terrain ont en ce sens été formelles à plusieurs reprises : « tontines et association, ça n'a rien à voir Madame! ». En France, les tontines se distinguent des initiatives associatives, contrairement à ce qui se passe en Afrique, où cette forme originale d'épargne et de crédits rotatifs a été gagnée ces trente dernières années par une vague de formalisation, du fait en partie de l'influence des ONG et des possibilités d'accès au micro crédit. Les tontines féminines en France se distinguent également des initiatives plus associatives des hommes dont l'objectif de valorisation de la mobilité des migrants à des fins de transformation villageoise est déclaré et fait l'objet de statuts (Quiminal, 2000). En France, les tontines féminines des femmes d'Afrique de l'Ouest, bien que légales, sont refoulées dans la sphère de l'invisible et les femmes entretiennent le mystère autour de ces pratiques, à l'égard des blancs, des administrations et des hommes. Durant cette première phase de l'enquête, la réalisation d'entretiens a cependant permis de constater un certain dynamisme de la pratique tontinière des femmes d'origine ouest africaines vivant en France, puisque presque toutes les femmes abordées dans le milieu associatif m'indiquaient faire par ailleurs partie d'une tontine. Mais il fut plus difficile de pénétrer la profondeur des réseaux et ce qui fait leur trame, la curiosité d'une Française attirant parfois plus de méfiance que d'intérêt. Les tontines relèvent d'une intimité difficilement accessible à Paris où l'ethnologie classique est un exercice difficile : il est épuisant d'établir des contacts quotidiens faute de lieux appropriés à la rencontre et au temps partagé. Les difficultés de communication peuvent également tenir à l'ambiguïté des positions respectives : dans les réseaux tontiniers de la parenté africaine étendue en France, qui des femmes africaines ou de l'ethnologue française occupe la position de l'étranger, et quelle norme culturelle d'accueil doit-on mutuellement se réserver? L'ethnologue qui enquête dans les réseaux immigrés peut se

5 En ceci notre méthode multisituée ne correspond pas strictement à la définition qu'en faisait Georges Marcus, qui différencie clairement comparatisme classique et ethnographie multisituée. L'ethnographie multisituée de l'auteur, plutôt appliquée aux cultural studies entend relier entre eux des éléments multisitués dont le rapprochement ne serait pas préalablement évident (Marcus, $1995: 8)$. 
trouver en position d'étranger, et en France faire parfois, par un jeu complexe d'acculturation ou de miroirs, les frais d'une certaine inhospitalité due à l'étranger. Ses informateurs se trouvent eux-mêmes dans une position ambivalente entre accueillis et accueillants, d'autant qu'ils peuvent aussi être devenus méfiants vis-à-vis notamment des enquêtes policières ou administratives peu bienveillantes à leur égard. Pour l'ethnologue, le fait d'avoir été préalablement intégré par la communauté d'origine représente alors un gage important.

Dans une deuxième phase d'enquête, il a donc été plus facile pour moi, bien que non réellement prémédité, de partir de la connaissance d'une parentèle khassonké ${ }^{6}$ au Mali pour être acceptée dans les réseaux tontiniers de cette communauté en France. C'est ainsi que m'est apparu l'intérêt méthodologique de suivre des pistes relationnelles au sein d'une communauté transnationale. La relation de confiance s'établit réellement à travers des allers-retours, dans une interaction avec des membres du réseau ici et là-bas et par l'intégration à un réseau d'interconnaissance et d'inter-reconnaissance délocalisée. Dans un contexte d'internationalisation, la notion contemporaine de localité devient plus une relation d'appartenance à un groupe ${ }^{7}$, et, dans les réseaux migrants ouest africains, ces groupes restent liés par une forte interconnaissance. Celle-ci peut d'ailleurs s'avérer réelle ou nominative ; certains membres du réseau de la parenté khassonké, évoquée ici, peuvent par exemple connaître juste de nom une tante qui a migré et vice-versa. Il s'agit pour l'ethnologue d'occuper une position dans un réseau de relation, position attribuée par la communauté non plus locale mais transnationale. On peut parler d'une « assignation » (Fogel, 2009) sur le terrain transnational, ce qui consiste à se voir accepté, intégré ou adopté. Notons qu'en contexte africain, qu'il soit local ou transnational, cette assignation se fait souvent par un recours au registre discursif de la parenté, qui occupe une place importante dans la définition des rôles de chacun. Ainsi l'étranger se voit attribué un nom malien et se trouve affilié à un lignage, au sein duquel il occupe une place qui permettra aux autres membres de la communauté de l'identifier et de le reconnaître, en fonction de celle-ci.

\section{CIRCULATIONS SYMBOLIQUES : LA PORTÉE TRANSNATIO- NALE DES ÉCHANGES CÉRÉMONIELS}

Dans la pensée d'auteurs comme Arjun Appaduraï ou Marshall Sahlins, la déterritorialisation de la localité n'entraîne pas, comme le prédisent certaines visions catastro-

6 Les Khassonké sont originaires de la région du Khasso, qui s'étend au Nord depuis la naissance du fleuve Sénégal de la rencontre du Bakoy et du Bafing au Mali, dans la région de Kayes. Ce peuple, issu d'un clan de peulhs « mandinguisés », au XIVe siècle, partage une langue proche du bambara. Les Khassonké partagent également une histoire commune avec leurs voisins et parents soninké. Bien que peu étudiées et moins importantes que celles des halpulaar et des soninké, les migrations khassonké ont donné naissance à une transnationalité. On trouve en France par exemple l'Entente Culturelle des Khassonké de France, qui organise régulièrement des cérémonies en France et au Mali.

7 Au sujet de ce rapport à la localité comme appartenance à un groupe, lire la préface de Marc Abélès pour la traduction en français du livre d'Arjun Appaduraï (2001). 
phiques de la mondialisation, une perte inéluctable des rituels et symboles, producteurs de sens et associés traditionnellement au quotidien partagé localement. Dans le contexte actuel, les symboles et rituels circulent au fil des réseaux de mobilité transnationale et de communication. L'ethnographie multi située est ethnographique en ce sens qu'elle permet d'observer la circulation des biens, symboles et rituels qui configurent des réseaux de relations vécues donnant corps à une communauté transnationale. Le réseau délocalisé devient construit comme un terrain, initial mais non donné à voir, au sein duquel il faut savoir s'intégrer pour observer le sens des gestes.

Les associations villageoises et les investissements dans leurs pays d'origine des Gens du Fleuve Sénégal, en particulier des Soninké et des Halpulaaren, ont été abondamment documentés ces dernières années (Quiminal, 1991 ; Lavigne Delville, 1991 ; Daum, 1998 ; Kane, 2002). Ces travaux ont permis de montrer que les interconnexions hors du village d'origine des sociétés d'aide mutuelle ont donné à ces associations un caractère et un dynamisme transnationaux. Du côté des femmes cependant, une focalisation sur les investissements associatifs en France a pu laisser croire qu'elles étaient moins portées à l'entretien du lien transnational. En effet, à travers l'engagement associatif, les femmes originaires d'Afrique de l'Ouest implantées en France ont principalement pour but de résoudre les problèmes de la vie quotidienne, elles procèdent de l'appropriation symbolique et pratique des territoires urbains en France (Quiminal, 2000) ${ }^{8}$. Pourtant une observation de la pratique tontinière de ces mêmes femmes migrantes au sein des réseaux informels de parenté donne à voir autre chose de leur relation aux communautés d'origine.

La tontine dite « des Kayésiennes Khassonké », par exemple, existe à Paris depuis 14 ans : 20 femmes, majoritairement originaires du quartier khassonké de la ville de Kayes au Mali, leurs parentes et leurs amies, se réunissent un samedi par mois pour rassembler une somme remise à tour de rôle à chacune des membres et qu'elles choisissent le plus souvent d'investir dans la construction d'une maison au pays ${ }^{9}$. En plus de cette cotisation, elles se soutiennent et s'investissent mutuellement dans les cérémonies familiales des unes et des autres entre la France et le Mali. Une cotisation de 20 euros par personne est prévue en cas de cérémonie familiale (mariage ou baptême) dans laquelle une des femmes membres de la tontine serait impliquée de près par exemple en tant que mère ou marraine (denbadiala). Cette dernière se voit désignée au sein de la parentèle ou du réseau tontinier, par une prestation versée par la mère. Elle est de fait obligée d'assurer la réussite de la cérémonie en prenant en charge une bonne partie des frais, et en particulier les dons osten-

8 En ce sens les dénominations des associations de migrants sont en effet assez significatives : alors que pour les hommes, la référence au territoire d'origine est déterminante : «Association des ressortissants de Niogomera en France », les associations féminines, moins nombreuses, privilégient au contraire la proximité spatiale et le quartier avec une référence plus large à la nationalité d'origine, s'intitulant par exemple « Association des Femmes Maliennes de Montreuil ». À la Maison des Associations de Montreuil, on dénombrait en 2001, 42 associations d'immigrés africains œuvrant pour la solidarité, dont 26 sont gérées exclusivement par des hommes, et 9 par des femmes.

9 J'ai traité ailleurs des motivations proprement féminines qui animent ces investissements dans des projets immobiliers dans les pays d'origine et des tensions que ces choix peuvent générer au sein des familles transnationales (Semin, 2007). 
tatoires aux griots ${ }^{10}$ et à la parentèle alliée. Certaines femmes ont donc d'important frais à couvrir lors des cérémonies familiales et sont soutenues pour ce faire par les membres de la tontine ; ceci étant souvent d'ailleurs une des principales raisons d'être du groupement.

Le soutien collectif peut intervenir lors d'une cérémonie qui a lieu en France, mais aussi au pays. Oumou Ndiaye, par exemple, s'est rendue au Mali pour le baptême de la fille d'une co-épouse restée au pays, à laquelle cette dernière devait donner le prénom d'Oumou. La relation homonymique est très importante dans les sociétés d'Afrique de l'Ouest, le fait de donner le prénom de quelqu'un à un enfant est une marque de reconnaissance et de respect. Oumou Ndiaye se devait en retour de donner de nombreux cadeaux à la mère de l'enfant. À cette occasion, les membres de la tontine en France ont été solidaires et ont versé à Oumou une contribution financière de 400 euros. En outre, la griotte ${ }^{11}$ attitrée du groupe de tontine en France, en voyage au Mali, a été invitée à la cérémonie par Oumou Ndiaye du fait de leur appartenance commune à la tontine en France. Elle y a chanté les louanges et valorisé les qualités et investissements de Oumou Ndiaye, ainsi que ceux du groupe de la tontine en France et de Mariem Diallo. Mariem, également membre de la tontine à Paris, avait, en raison de son cousinage avec Oumou Ndiaye, fait « sacrifier un bœuf » pour la cérémonie, c'est-à-dire qu'elle avait envoyé depuis la France, et par la main de la griotte, une somme d'argent équivalent à l'achat d'une vache, dont la viande a été consommée pendant le baptême. À travers leurs dons et les paroles de la griotte, les femmes de la tontine étaient donc comme présentes à Kayes lors de la cérémonie du baptême, tout en restant physiquement en France, elles faisaient en quelque sorte de cette manière l'expérience d'une forme de présence simultanée.

Quelques mois après, lors d'un tour de tontine à Paris, la réussite de la cérémonie et la valeur du « sacrifice » ont été célébrées en retour à travers une séance de louanges, nous valant la présence exceptionnelle de l'époux de la griotte, lui-même griot renommé. Les tontines sont restées en France « affaires de femmes », bien distinctes de celles des hommes. La participation physique d'un homme adulte à une réunion de tontine féminine est rare, elle n'est possible que parce qu'il s'agit d'un griot et qu'il a quelque chose d'important à dire. À travers sa prestation, ce dernier a valorisé un certain type de comportement, celui qui consiste à accorder malgré la migration une importance primordiale aux dons cérémoniels dans une implication mutuelle des réseaux de l'amitié et de la parenté entre ici et là-bas. Dans le discours du griot, la dimension transnationale semblait grandir encore la valeur des gestes d'Oumou Ndiaye et de Mariem Diallo. La valeur différentielle des prix entre la France et l'Afrique permet déjà aux migrants de se distinguer par des dons cérémoniels importants, mais les paroles du griot visaient en l'occurrence à glorifier en particulier l'investissement symbolique dans sa dimension transnationale. Oumou Ndiaye

10 Les Khassonké sont organisés au sein d'une société dite à " castes » qui désigne, bien que le terme puisse être discuté, une répartition en groupe de métiers endogames. Parmi ceux-ci existe celui des griots : les artistes qui ont en charge la diffusion de l'histoire des familles et des hommes, transmis par la parole et la musique. Bien que de condition inférieure dans la hiérarchie sociale, les griots sont craints et respectés pour le pouvoir de leur verbe. On les paie pour leurs louanges, qui construisent la réputation des hommes et des femmes (Voir sur ce sujet : Camara, 1992 ; Conrad et Frank, 1995).

11 Une « griotte » est une femme qui appartient au groupe des griots et exerce les fonctions spécifiques relatives à ce groupe dans la communauté. 
a tout d'abord été remerciée pour avoir invité la griotte à représenter le groupe lors de cette cérémonie au Mali, la Présidente de la tontine en France a également été saluée pour avoir su gérer la tontine dans le respect des valeurs traditionnelles, ainsi que Mariem Diallo pour le sacrifice symbolique du bœuf (le tour avait lieu chez elle ce jour-là). L'émotion suscitée par la présence du griot dénotait la force de cette connexion symbolique entre deux sites. Cette observation multi située a été permise par une connaissance particulière du réseau de parenté et d'affinités entre ici et là-bas. Du fait d'une division sexuelle des tâches rituelles, les femmes sont plus investies dans ce type d'échanges cérémoniels, et leur participation active aux tontines est notamment liée à cette nécessité d'y assister physiquement, symboliquement et financièrement, notamment me semble-il pour entretenir l'inter-connaissance et la publicité des événements familiaux au sein de la communauté transnationale. Les griots comme porte-paroles et représentants peuvent alors jouer en ce sens un rôle transitionnel important dans le maintien des communautés par-delà les frontières, et leur rôle de communicateur s'adapte à la déterritorialisation des liens.

L'importance de l'aspect cérémoniel des échanges longue distance n'est pas une nouveauté, elle a été démontrée au sujet des échanges de coquillage dans « Les Argonautes du Pacifique occidental ». Mais, comme Bronislaw Malinowski pour les Iles Trobriands, dont les travaux ont été complétés par ceux d'Annette Weiner sur les échanges féminins, les africanistes et spécialistes de la migration ont un peu occulté le rôle des femmes dans la reproduction de l'espace transnational au profit des transferts économiques masculins. De fait on passe à côté d'une dimension intime des liens tissés par les échanges cérémoniels, et de la continuité des échanges au-delà de la migration qui fait aussi une force des diasporas africaines. Les hommes migrants ont un rôle à jouer dans ce dynamisme, et ce sont eux qui sont les plus sollicités depuis le Mali pour financer les prestations matrimoniales et en particulier la dot des jeunes et moins jeunes, réinvesties ensuite par les femmes dans les échanges cérémoniels. Ceci s'inscrit d'ailleurs dans une certaine continuité avec les migrations saisonnières qui visaient souvent à satisfaire les exigences de l'alliance.

Il est possible, comme Alejandro Portes le propose, de comprendre l'idée de communauté et de flux transnationaux comme des « formes originales et potentiellement puissantes de mondialisation par le bas », qui tirant parti du différentiel des prix entre les continents s'inscrivent dans la logique même du capitalisme (Portes, 1999). L'appropriation des modes occidentaux de production et de consommation apparaît d'ailleurs souvent dans les processus de globalisation, dont celui de la migration, comme une inévitable occidentalisation du monde. Les études qui concluent à une « orientalisation de l'Ouest» (MacLuhan cité par Hylland Eriksen, 2003) ou une " indigénisation de la modernité » (Sahlins, 1997 : 17) sont moins nombreuses. L'un et l'autre processus ne sont d'ailleurs pas mutuellement exclusifs, comme l'affirmait Deleuze dans sa définition du rhizome : « il n'y a pas de capitalisme universel et en soi le capitalisme est au croisement de toutes sortes de formations » (Deleuze, $1980: 30$ ), et la focalisation sur l'occidentalisation est peut-être due à un défaut d'intégration des chercheurs au sein de ses réseaux, intégration permettant le décentrement. Malinowski se désolait dans l'introduction des Argonautes du Pacifique de ce que l'objet d'étude des ethnologues, à savoir les cultures locales et l'échange symbolique, avait tendance à fondre à peine ces derniers avaient-ils fait leurs armes. On pourrait lui répondre avec Sahlins et à la lumière des phénomènes contemporains que l'observation et la mise en exergue de la cohérence de dons symboliques est toujours d'actualité et 
mérite plus d'attention. L'ethnographie multi située, en tant qu'exercice d'intégration au sein des communautés transnationales, permet d'observer les modes de connexion et de renouvellement des relations avec le pays d'origine, malgré la distance. Les communautés transnationales sont à l'évidence structurées par l'échange économique, social, et j'ajouterais donc cérémoniel.

On peut s'interroger sur l'avenir des remises monétaires et des projets de co-développement compte tenu du vieillissement des immigrés africains, de la fermeture des frontières, et des motivations différentes des jeunes issus de l'immigration (Kane, 2002 ; Dia, 2008). La force et la longévité de ces liens réticulaires est donc en question aujourd'hui. Les caisses villageoises tentent avec plus ou moins de succès d'associer la deuxième génération à leurs efforts pour le développement sanitaire et social des communautés d'origine (Kane, 2002 : 261). Il est intéressant par exemple de voir comment les mères tentent de même de transmettre, à travers notamment les tontines, le geste de l'investissement et de l'implication cérémonielle à leurs filles.

\section{CONCLUSION}

La méthode de l'ethnographie multi située, comprise comme un ensemble d'observations participantes réalisées au sein d'un réseau de relations dont les ancrages sont spatialement distants, correspond donc à une adaptation méthodologique de l'ethnographie à la déterritorialisation des rapports sociaux et symboliques. Elle ne remet pas en cause la nécessité d'une intégration pour une observation de l'intérieur. En ce sens le positionnement nécessaire à l'ethnographie multi située ne diffère pas fondamentalement de celui nécessaire à l'ethnographie classique, de même que les modes de liens à observer restent approximativement les mêmes qu'ils soient ou non territorialisés. Dans l'observation de l'échange au sein des communautés transnationales, on interroge le réseau comme terrain, préexistant bien que non donné à voir. La méthode multi située de l'ethnographie réside donc dans la multiplication des échelles spatiales du terrain, ce qui ajoute une forme de complexité.

Le changement social contemporain réside dans le changement des modes de reproduction sociale, territoriale et culturelle des communautés. Grâce au développement des migrations et des télécommunications, l'identité de groupe se développe aujourd'hui en référence à des territoires et des systèmes de représentation multiples. Aujourd'hui, il s'agit donc d'envisager l'enquête de terrain comme une recherche épousant au plus près les contours transnationaux des paysages référentiels et relationnels. La méthode multi située, bien qu'elle s'impose souvent d'elle-même dans les études migratoires, est souvent considérée comme une marque de superficialité par les anthropologues, partisans de la profondeur des terrains longs et statiques, permettant une intégration relative. L'idéal serait de pouvoir conserver la profondeur de la micro enquête au plus près des réalités vécues, tout en l'intégrant à des cadres spatio-temporels élargis. Au sein des communautés transnationales, l'intégration est permise par le mouvement, et nécessaire à l'observation du sens des échanges déterritorialisés. 


\section{Références bibliographiques}

AMSELLE Jean-Loup (2001) Branchements : Anthropologie de l'universalité des cultures, Paris, Flammarion, $233 \mathrm{p}$.

APPADURAÏ Arjun (2001) Après le colonialisme. Les conséquences culturelles de la globalisation, (traduction de Modernity at Large: Cultural Dimensions of Globalization, 1996) Paris, Payot, $322 \mathrm{p}$.

APPADURAÏ Arjun (1991) Global ethnoscapes. Notes and queries for a transnational anthropology, in Richard Fox Ed., Recapturing anthropology. Working in the present, School of American Research Press, Santa Fe, New Mexico, pp. 190-210.

BAUMAN Zygmunt (2007) Le présent liquide, Paris, éditions du seuil, 143 p.

BERTHOMIERE William et HILY Marie-Antoinette (2006), Décrire les migrations internationales. Les expériences de la co-présence, Vingtième anniversaire. Revue Européenne des Migrations Internationales, vol. 22, $\mathrm{n}^{\circ} 2$, pp. 67-82.

BOESEN Elisabeth et MARFAING Laurence Dir. (2007) Les nouveaux urbains dans l'espace Sahara-Sahel. Un cosmopolitisme par le bas, Paris, Karthala, 330 p.

BOURDIEU Pierre (2000) L'objectivation participante. Discours prononcé lors de la remise de la Huxley Memorial Medal, The Journal of the Royal Anthropological Institute, juin 2003, pp. 282-294.

BRACHET Julien (2009) Géographie du mouvement, géographie en mouvement. La mobilité comme dimension du terrain d'étude dans les migrations, www.hal.archiveouvertes.fr/ docs $/ 00 / 35 / . . . / \mathrm{PDF} /$ brachet_terrain_arras.pdf

BRETTELL Caroline (2000) Theorizing Migration in Anthropology, in C. Brettell and J. Hollifield, Migration Theory. Talking across disciplines, New York, Routledge, pp. 97-135.

BRYCESSON Deborah and VUORELA Ulla Eds (2002) The transnational family, Oxford-NewYork, Berg, $267 \mathrm{p}$.

CAILLE Alain (2007) Anthropologie du don. Le tiers paradigme, La découverte, Paris, 280 p.

CAMARA Sory (1992) Gens de la parole. Essai sur la condition et le rôle des griots dans la société malinké, Paris, Karthala, 356 p.

CONRAD David and FRANK Barbara Eds. (1995) Status and Identity in West Africa. Nyamakalas of Mande, Indiana Press University, Indianapolis, $191 \mathrm{p}$.

DAUM Christophe (1998) Les associations de Maliens en France, Paris, Karthala, 253 p.

DELEUZE Gilles et GUATARI Félix (1980) Milles Plateaux. Capitalisme et schizophrénie, Éditions de Minuit, $640 \mathrm{p}$.

DIA Hamidou (2007) Les investissements des migrants dans la Vallée du Fleuve Sénégal : confiance et conflits d'intérêt, Revue Européenne des Migrations Internationales, vol. 23, n 3, pp. 29-49.

DIA Hamidou (2008) Villages multi-situés du Fouta-Toro en France : le défi de la transition entre générations de caissiers, lettrés et citadins, in E. Bouilly et N. Marx, Migrations et Sénégal, Asylons, $\mathrm{n}^{\circ} 3$, http://www.reseau-terra.eu/article713.html, site consulté le 12 novembre 2009.

DUMONT Gérard François (2007) Les nouvelles technologies migratoires au XXIe siècle, Revue française de géopolitique, $\mathrm{n}^{\circ} 17, \mathrm{pp}$ 47-62.

FOGEL Frédérique (2009) L'ethnologue et l'assignation, sur le terrain de la parenté, La relation ethnographique, terrains et textes. Mélanges offerts à Raymond Jamous, Laboratoire d'ethnologie et de sociologie comparative, http://ateliers.revues.org/document8213.html, site consulté le 12 novembre 2009.

GHASSARIAN Christian Éd. (2002) De l'ethnographie à l'anthropologie réflexive. Nouveaux terrains, nouvelles pratiques, nouveaux enjeux, Armand Colin, Paris, 249 p.

GLICK SCHILLER Nina, BASCH Linda and BLANC-SZANTON Christina (1992) Towards a transnationalization of migration: race, class, ethnicity and nationalism reconsidered, in The Annals of the New York Academy of Sciences, 645, New York, New York Academy of Sciences. 
HYLLAND ERIKSEN Thomas Éd. (2003) Globalisation: Studies in Anthropology, London, Pluto, $226 \mathrm{p}$.

KANE Abdoulaye (2001) Les caméléons de la finance populaire au Sénégal et dans la diaspora. Dynamique des tontines et des caisses villageoise entre Thilogne, Dakar et la France, Thèse de l'Université d'Amsterdam, $200 \mathrm{p}$.

LAVIGNE DELVILLE Philippe (1991) Migration et structuration associative. Enjeux dans la moyenne vallée du fleuve Sénégal, in Bernard Crousse, Philippe Mathieu et Sidi Mohammed Seck Éds, La Vallée du fleuve Sénégal. Évaluations et perspectives d'une décennie d'aménagement, Paris, Karthala, 384 p.

LESERVOISIER Olivier (2005) L'anthropologie réflexive comme exigence méthodologique et épistémologique, Paris, Karthala, 327 p.

MA MUNG Emmanuel (2000) La Diaspora chinoise, géographie d'une migration, Paris, Ophrys, $176 \mathrm{p}$.

MARCUS George E. (1995) Ethnography in/of the World System: The emergence of multi-sited Ethnography, Annual review of anthropology, 24, pp. 95-117.

MARCUS George E. (1998) Ethnography through, thick and thin, Princeton, University Press, $248 \mathrm{p}$.

MERCKLE Pierre (2008) Sociologie des réseaux sociaux, Paris, La découverte, 105 p.

MARIE Alain (1997) L'Afrique des individus. Paris, Karthala, 436 p.

PORTES Alejandro (1999) La mondialisation par le bas, l'émergence de communautés transnationales, Actes de la recherche en sciences sociales, $\mathrm{n}^{\circ} 129$, pp. 15-25.

QUIMINAL Catherine (1991) Gens d'ici, gens d'ailleurs, Paris, Christian Bourgeois, 222 p.

QUIMINAL Catherine (1997) Un réseau d'associations de femmes africaines, Migrations en tout genre, Hommes et Migrations, $\mathrm{n}^{\circ}$ 1208, pp. 24-30.

QUIMINAL Catherine (2000) Construction des identités en situation migratoire : territoire des hommes, territoires des femmes, Autrepart, n 14, pp. 107-120.

SAHLINS Marshall (2001) Les Lumières en anthropologie?, Nanterre, Société d'Ethnologie, 40 p.

SAYAD Abdelmalek (1999) La double absence, des illusions de l'émigré aux souffrances de l'immigré, Éditions du Seuil, Paris, 448 p.

SEMIN Jeanne (2007) L'argent, la famille et les amies. Ethnographie contemporaine des tontines africaines en contexte migratoire, Civilisations, vol. LVI (1-2), Bruxelles, ULB, pp. 183-199.

SCHMITZ Jean et HUMERY Marie-Eve (2008) La Vallée du Sénégal entre (co)développement et transnationalisme. Irrigation, alphabétisation et migration ou les illusions perdues, Politique africaine, $\mathrm{n}^{\circ} 109$, pp. 56-73.

TARRIUS Alain (2002) La mondialisation par le bas. Les nouveaux nomades de l'économie Souterraine, Paris, Balland, $168 \mathrm{p}$.

WEINER Annette (1983) La richesse des femmes ou comment l'esprit vient aux hommes, Paris, Éditions du Seuil, 280 p. 


\title{
L'ethnologue dans les réseaux économiques des femmes migrantes : modes de présence simultanée entre la France et l'Afrique
}

\author{
Jeanne SEMIN
}

\begin{abstract}
À travers une réflexion autour du concept d'ethnographie multi située, l'auteur se propose de redéfinir l'éclairage spécifique que peut apporter une démarche de type ethnographique à l'étude des migrations. Elle s'interroge autant sur l'actualité de la notion de territoire que sur la pertinence de l'idée d'un renouvellement méthodologique nécessaire à l'observation des sociétés dites postcoloniales. Sa question principale demeure de savoir comment observer, au sein des communautés transnationales, le lien entre des individus proches, mais que l'espace sépare. À partir d'une intégration au fil des réseaux féminins d'immigrés khassonkés en France, elle livre un exemple d'observation multi située et introduit la dimension cérémonielle des échanges dans la caractérisation du lien transnational.
\end{abstract}

\section{An Ethnologist in the Economic Networks of Migrant Women: Simultaneous Ways of Moving between France and Africa}

\author{
Jeanne SEMIN
}

Re-thinking the concept of multi-located ethnography, the author aims at redefining the specific light shed by an ethnographic approach of the study of migrations. She questions the actuality of the notion of territory as well as the relevance of the notion of methodological renewal necessary for observing so-called post colonial societies. Her main point is to find out how to observe, within trans-national communities, the links between individuals, close to one another, but who are pulled apart in space. As she became integrated into networks of Khassonké women immigrants in France, she provided an example of multi-located observation, and introduced the ceremonial dimension of exchanges to characterize the trans-national links.

\section{Una etnóloga en las redes económicas de mujeres migrantes: modos de presencia simultánea entre Francia y África}

\author{
Jeanne SEMIN
}

Reflexionando acerca del concepto de etnografía multi localizada, la autora se propone de volver a definir el punto de vista específico que puede aportar al estudio de las migraciones la investigación de tipo etnográfico. Se interroga tanto sobre la actualidad de la noción de territorio como sobre la pertinencia de la necesidad de una renovación metodológica para observar las sociedades llamadas postcoloniales. Su preocupación principal sigue siendo como observar, en las comunidades transnacionales, el lazo entre individuos cercanos pero que el espacio separa. Integrándose en redes femeninas de inmigrantes khassonkes en Francia, nos ofrece un ejemplo de observación multi situada e introduce la dimensión ceremonial de los intercambios en la caracterización del lazo transnacional. 